\title{
Study of optimum shape and strength design of the redesigned Zig-Zag chair
}

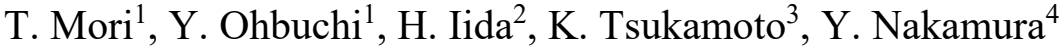 \\ \& H. Sakamoto ${ }^{1}$ \\ ${ }^{1}$ Graduate School of Advance Science Technology Research, \\ Kumamoto University, Japan \\ ${ }^{2}$ Sojo University, Japan \\ ${ }^{3}$ National Institute of Technology, Kagoshima College, Japan \\ ${ }^{4}$ Osaka Sangyo University, Japan
}

\begin{abstract}
Existing product developments tend to separate the product design from the product planning. After the planning of shape and style of the product by designer, its strength is evaluated by engineering analysis, and then the product will be finally designed.

This research, aims at the construction of the optimum shape and strength with the sophisticated design of the machine and structure members. The composite material that are typified by carbon fiber reinforced plastic (CFRP) has very high specific strength and it is light compared with metals. So, it is possible to produce the complex and lightweight products by using these composite materials.

Here, the material characteristics were expressed as a combination of some simple element models of this high strength carbon fiber reinforced materials CFRP. By the simulation design technique which used the FEM analysis was proposed. To get the elastic modulus of CFRP which is influenced by fiber direction, three-point bending test was performed. In this experiment, two types of specimens which have different fiber direction each other were tested.

As a design target, the redesign of Zig-Zag chair was selected, which was designed by Gerrit Thomas Rietveld, who was a Dutch architect and furniture designer. The optimum design items are the good shape, best strength and weight lightening.

Keywords: CFRP, Zig-Zag chair, elastic modulus, torsional rigidity.
\end{abstract}




\section{Introduction}

In general product development, there are many designs which have difficulty to commercialize from a restriction of strength and weight, even if they have wonderful shape and function. This is because in conventional product development, product design tends to separate from product planning. After planning of shape and style of product by designer, its strength is evaluated by engineering analysis, and then the product will be finally designed $[1,2]$. In this study, CFRP which is composite material and has very high specific strength and elastic modulus is targeted. We examine its material characteristic, and pursue the optimum structure design by using its material characteristic effectively [3-5]. Development of new product design method integrated with strength evaluation and styling enables them to be commercialized.

\section{Subject of study}

The subject of this study is the Zig-Zag chair (shown in Fig. 1) [5]. It was designed in 1934 by Gerrit Thomas Rietvelt who was a Dutch architect and cabinet manufacturer. This chair is formed by four straight-line wooden panels which consist of a backrest, seat, chair support and floor support. They are supported by a small corner block (reinforcing member). This chair has enough strength despite its appearance, and its design quality also has been assessed highly. However, the original wooden structure is $7.0 \mathrm{~kg}$. It's too heavy to handle as a usual chair.

The Zig-Zag chair was redesigned in 2015 (Fig. 2) by Haruhiko Iida [5-7]. It was made of CFRP with some folds for reinforcement, and was drastically lightened. The weight is only $3.5 \mathrm{~kg}$; very light compared with the original ZigZag chair. This redesigned Zig-Zag chair has sufficient strength for sitting, but it has a problem. When using the redesigned Zig-Zag chair, torsional deformation is felt, and it makes the user uneasy. Therefore, we pursue the optimum shape of the Zig-Zag chair to have sufficient torsional rigidity in this study.

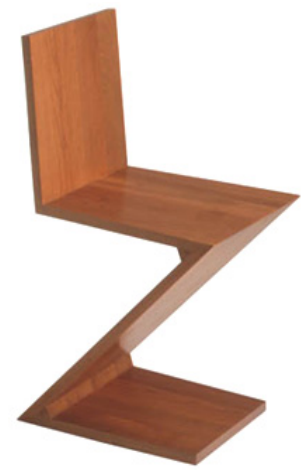

Figure 1: Original Zig-Zag chair.

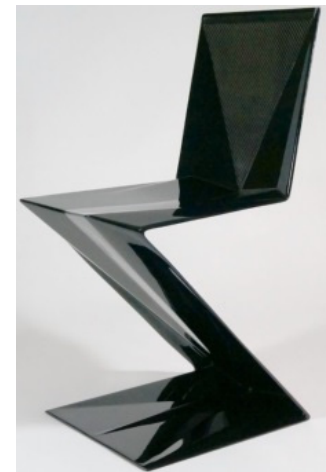

Figure 2: Redesigned Zig-Zag chair. 


\section{Three-point bending test}

To get the basic material characteristic of CFRP which has anisotropy, three-point bending test was performed. In this experiment, two types of specimens which have different fiber direction of plain weave CFRP cloth were tested. Specimen types are as follows, (a); its fiber direction to longitudinal direction of specimen is 0 deg., (b); its fiber direction to longitudinal direction of specimen is $45 \mathrm{deg}$. The number of lamination is 8ply. The dimensions of specimen are as follows, the length is $100 \mathrm{~mm}$, the width is $16 \mathrm{~mm}$ and the thickness is $2.6 \mathrm{~mm}$. Its schematic sketch is shown in Fig. 3. The experimental result of the three-point bending test is shown in Table 1. It is clear that a remarkable anisotropy of CFRP appears.

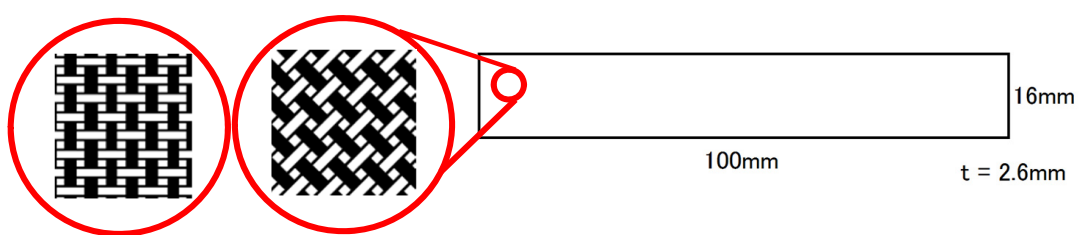

(a)

(b)

Figure 3: Schematic sketch and dimensions of specimen.

Table 1: Result of the three-point bending test.

\begin{tabular}{|c|c|c|}
\hline Direction of cloth (deg.) & 0 & 45 \\
\hline Elastic modulus (GPa) & 33.3 & 9.32 \\
\hline
\end{tabular}

\section{Representation of CFRP cloth in CAD modelling}

In analysing the structure mode of CFRP, it is necessary to represent the characteristics of CFRP on CAE analysis. The characteristic is elastic modulus along the fiber direction are high, and along the other directions are low. The modeling method of CFRP structure to represent the result of previous three-point bending test on CAD modeling well must be proposed. The sketch of actual plain weave fiber cloth is shown on the left side of Fig. 4. In this study, it is represented by a pair of unidirectional layers which are laminated orthogonally, as shown on the right side of Fig. 4. 

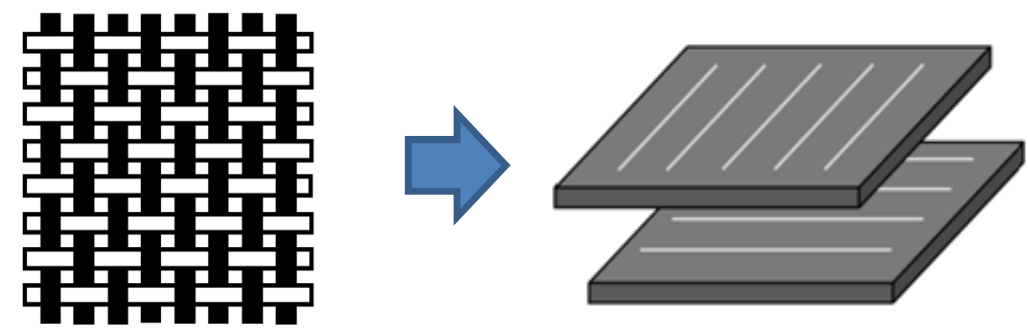

Figure 4: Representation of the plain weave CFRP cloth.

The elastic modulus of one layer unidirectional CFRP (right side of Fig. 4) in the direction of fiber is estimated by theory of composite material of equation (1).

$$
E_{\text {comp }}=E_{f} V_{f}+E_{m}\left(1-V_{f}\right)
$$

Here, $E_{c o m p}, E_{f}, E_{m}$ are elastic modulus of composite material, fibers and resin respectively and $V_{f}$ is volume ratio of fibers. From the characteristic of CFRP, since the elastic modulus of resin is predominant for the direction orthogonal to the fiber direction, the elastic modulus of direction orthogonal to the fiber direction was assumed to be $3.0 \mathrm{GPa}$ which is the value of resin. The volume of fiber in a longitudinal direction and orthogonal direction is assumed to be $50 \%$ respectively. The result of a previous three-point bending test is substituted in $E_{\text {comp }}$, and then the value of $E_{f}$ was estimated to be $63.6 \mathrm{GPa}$.

Using this value, a three-point bending test simulation was performed with the CAD model of the CFRP structure described above. The simulation was performed for two fiber directions, 0 and $45 \mathrm{deg}$. the same as an actual three-point bending test. From the result of this simulation and eq. (2) for maximum deflection in the three-point bending, elastic modulus of the model that represents CFRP made of plain weave cloth can be calculated.

$$
y_{\max }=\frac{W l^{3}}{48 E I_{z}}
$$

Here, $W$ is load, $l$ is a distance between supporting points, $E$ is elastic modulus, $I_{Z}$ is geometrical moment of inertia. The result of simulation is shown in Table 2.

Table 2: Result of bending test simulation.

\begin{tabular}{|c|c|c|}
\hline Direction of cloth (deg.) & 0 & 45 \\
\hline Elastic modulus $(\mathrm{GPa})$ & 32.8 & 9.68 \\
\hline
\end{tabular}

As a result, the elastic modulus of simulation result in both directions is close to the result value of the actual three-point bending test. For improvement of 
further accuracy of proposed model, the elastic modulus of parallel to fiber direction of single layer CFRP (call X-direction below), and of orthogonal to fiber direction (call Y-direction below), were gradually changed and simulation was performed. Figs 5 and 6 show the effects of elastic modulus of $\mathrm{X}$ and $\mathrm{Y}$-directions to elastic modulus of specimen with fiber direction of 0 and $45 \mathrm{deg}$. CFRP cloth model. As shown in Fig. 5, the elastic modulus of X-direction increases, the elastic modulus of specimen with $0 \mathrm{deg}$. fiber direction increases. Therefore, the elastic modulus in $0 \mathrm{deg}$. fiber direction can be tuned. On the other hand, by changing of elastic modulus of Y-direction, the elastic modulus in 45 deg. fiber direction can be tuned. Finally the elastic modulus values became almost equal to the experimental result by using the tuned values shown in Table 3.

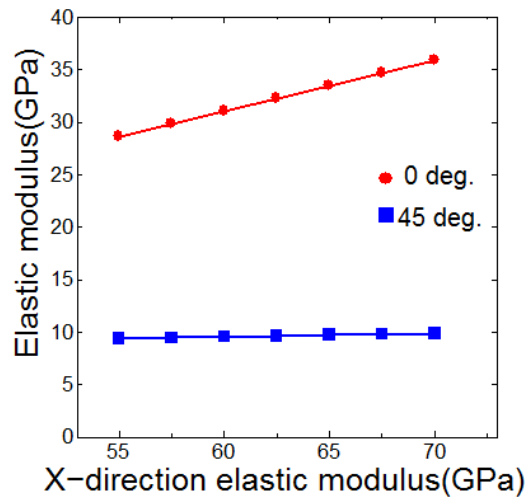

Figure 5: Effect of X-direction (elastic modulus).

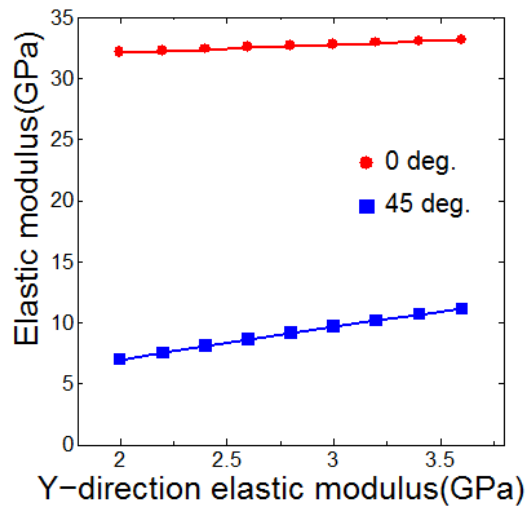

Figure 6: Effect of Y-direction (elastic modulus).

Table 3: Elastic modulus of single layer CFRP and result of bending test.

\begin{tabular}{|c|c|c|c|c|}
\hline & \multicolumn{2}{|c|}{$\begin{array}{c}\text { Elastic modulus of } \\
\text { single layer CFRP }(\mathrm{GPa})\end{array}$} & \multicolumn{2}{|c|}{$\begin{array}{c}\text { Elastic modulus of CFRP } \\
(\mathrm{GPa})\end{array}$} \\
\hline & \multicolumn{2}{|c|}{ Direction of fibers } & Direction of cloth (deg.) \\
\hline Direction & $\mathrm{X}$ & $\mathrm{Y}$ & 0 & 45 \\
\hline First assumption & 63.6 & 3.0 & 32.8 & 9.48 \\
\hline Tuned value (final) & 65.0 & 2.84 & 33.3 & 9.32 \\
\hline
\end{tabular}

To confirm validity of the analysis examined above, the maximum stress of each layer of the specimen in three-point bending simulation is shown in Fig. 7. In this simulation, fiber direction in layers of odd number is parallel to longitudinal direction, and layers of even number are orthogonal to longitudinal direction. Therefore, the layers of odd number show large stress, and the layers of even number show small stress as shown here. But, in the actual CFRP structure using cloth, these stresses will indicate average value. Since the simplified CFRP structure model well represent stress distribution in bending test, proposed model of CFRP cloth has validity. 


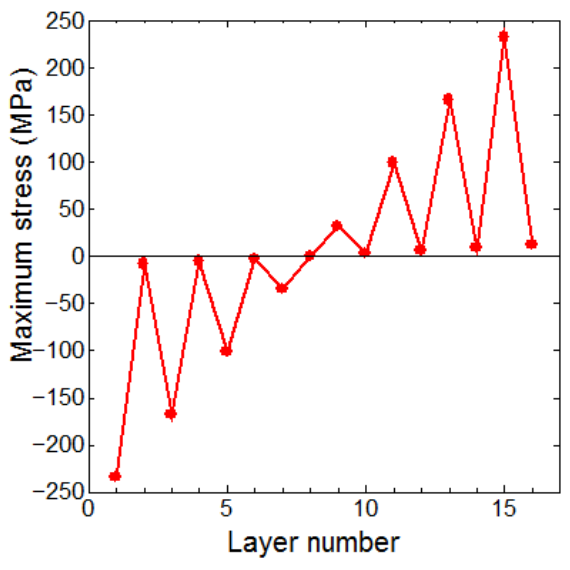

Figure 7: Maximum stress in each layer.

\section{Torsional rigidity of redesigned Zig-Zag chair}

When using the redesigned Zig-Zag chair, torsional deformation is felt in the case of biased load and it makes the user uneasy. Above-mentioned representation of CFRP cloth is applied to redesigned Zig-Zag chair and simulation is performed, and improving the torsional rigidity is attempted.

Here, various structures using above mentioned CFRP model are proposed, and simulation is performed to improve torsional rigidity. The CAD model of redesigned Zig-Zag chair made of CFRP with fold structure is shown in Fig. 8. As the first attempt to improve the torsional rigidity, the leg part is changed to box structure. Here, simplified box structure model with a few folds is used because it is very difficult to make box structure keeping the shape of complex fold structure as shown in Fig. 8. The simplified model is without back, its seat and floor support are flat with high rigidity, and the fold of leg is simplified as shown in Fig. 9. Using this model, simulation is performed changing size of space of box structure, and improvement of torsional rigidity is examined. Loading condition of this simulation is shown in Fig. 10. Fig. 11 shows rotation of seat. The floor support plane is fixed and distributed load is applied to front-right quarter area of seat. Here, space of box structure is defined as t shown in Fig. 9, and rotational angle of seat shown in Fig. 11 is defined as $\theta$. The simulation result is shown in Fig. 12. 


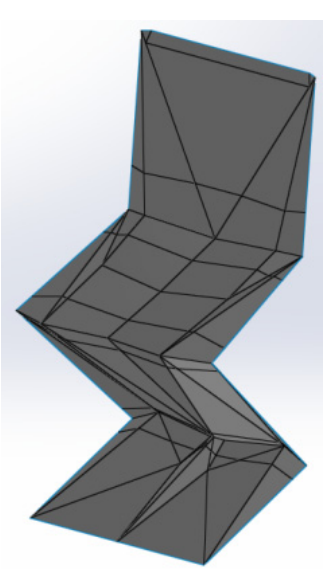

Figure 8: Zig-Zag chair constructed in CAD modeling.

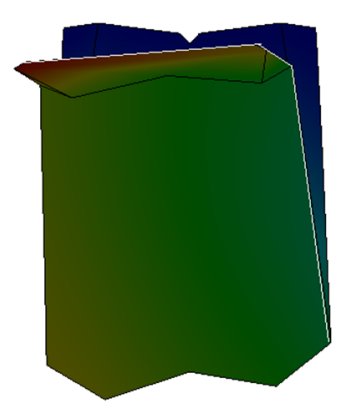

Figure 11: Rotation of seat.

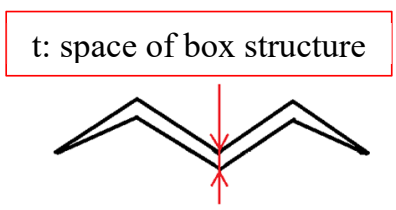

Cross section of leg

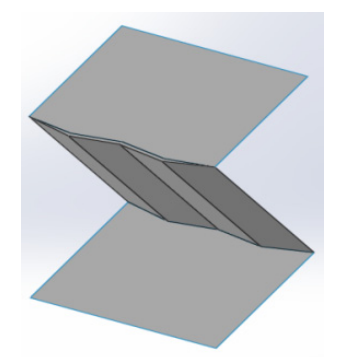

Figure 9: Simplified model.

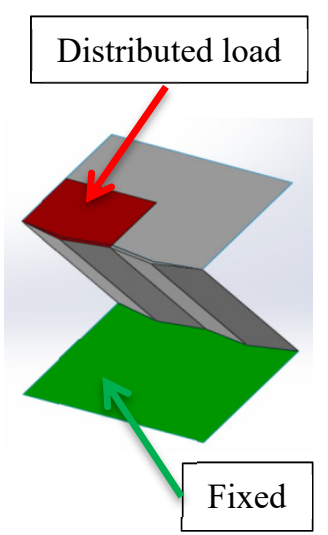

Figure 10: Analysis condition.

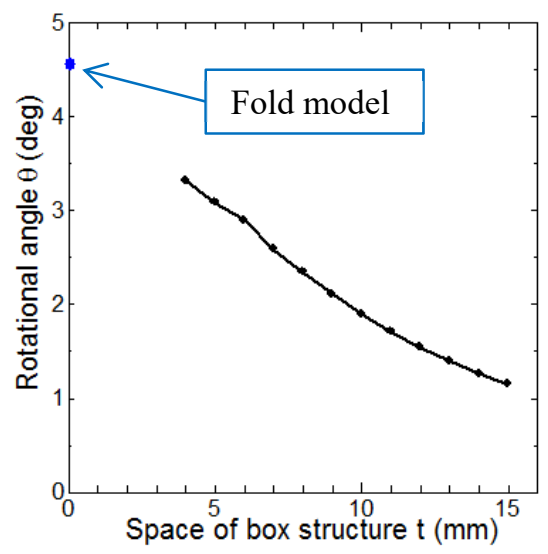

Figure 12: Suppression of rotation by box structure.

As shown in this figure, torsional rigidity of Zig-Zag chair is improved with increasing space of box structure. Using $15 \mathrm{~mm}$ space size, the rigidity quadruples compared with the fold structure. However, the size of space should be decided from both strength and styling viewpoints for the design of such product. The larger size of box space will change the excellent shape of this designed chair. 
Also, it is necessary to consider about difficulty of product making process of CFRP, because box structure with fold shape might be too complex for mass production.

Then, as a next attempt, the influence of fiber direction of CFRP cloth on torsional rigidity is examined. The rigidity is examined by simulation using proposed CFRP model, changing of fiber direction of cloth. The simulation is performed using simple model (Fig. 9) for two cases, one is for the fiber direction of 0 deg. (Fig. 13(a)), and the other is 45 deg. (Fig. 13(b)).

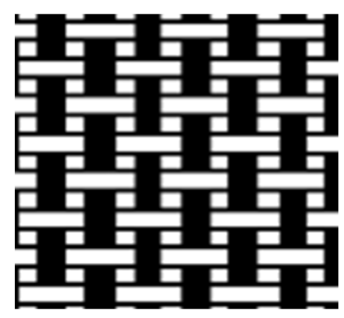

(a) 0 deg.

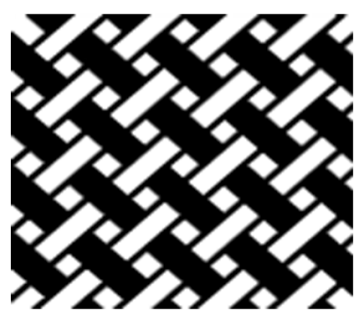

(b) $45 \mathrm{deg}$.

Figure 13: Fiber direction in the leg part of Zig-Zag chair.

The results are shown in Figs 14 and 15. Here, vertical displacement of the seat is defined as $U_{z}$. From the result of Fig. 14, the model of $45 \mathrm{deg}$. fiber directions has higher torsional rigidity than of $0 \mathrm{deg}$. fiber direction. On the other hand, the model with $0 \mathrm{deg}$. fiber direction shows higher rigidity in vertical direction than the model with a $45 \mathrm{deg}$. fiber direction which shows higher

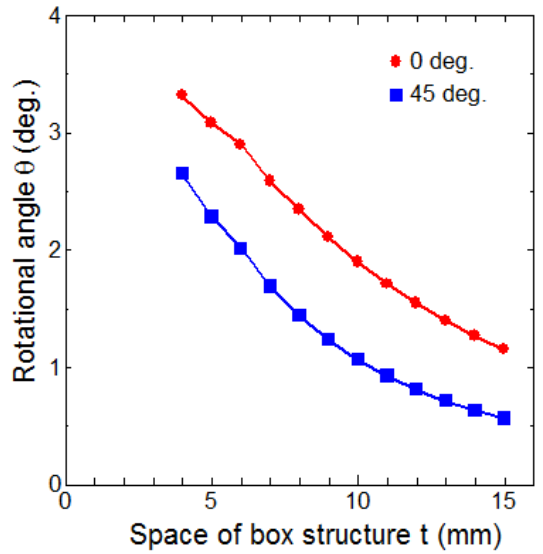

Figure 14: Rotation of seat comparing 0 and 45 deg.

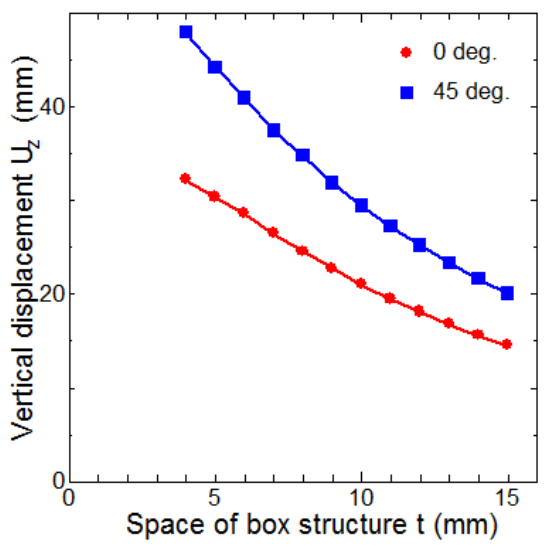

Figure 15: Vertical displacement comparing 0 and $45 \mathrm{deg}$. 
torsional rigidity (as shown in Fig. 15). However, the vertical displacement can be expected to improve sitting feelings. Therefore, the use of a $45 \mathrm{deg}$. fiber direction cloth is expected to be a suitable CFRP structure for the chair with high torsional rigidity and high vertical flexibility.

As the third proposal, the influence of fiber angle in CFRP cloth mesh is examined in simulation using the fold structure model of Zig-Zag chair (Fig. 8).

In this simulation, fiber angle of CFRP cloth mesh is changed bisymmetrically from 0 to 90 deg. by $10 \mathrm{deg}$. (as shown in Fig. 16).

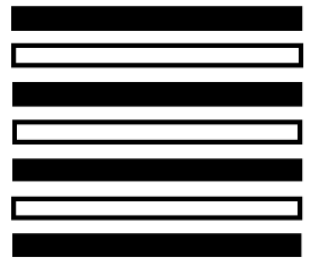

$0 \mathrm{deg}$.

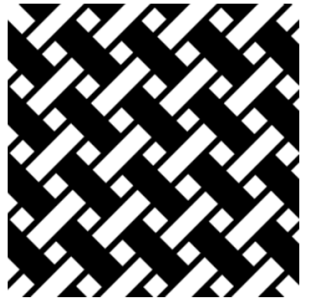

45 deg.

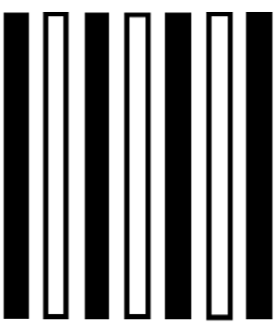

90 deg.

Figure 16: Fiber angle in cloth changed from 0 to 90 deg. bisymmetrically.

The results are shown in Figs 17 . This figure shows that torsional rigidity is highest when the fiber angle is near $50 \mathrm{deg}$., and vertical displacement is smallest when the fiber angle is near $60 \mathrm{deg}$. From this result, both rotational distortion and vertical displacement of Zig-Zag chair can be restrained when fiber angle is 50 to $60 \mathrm{deg}$. So it is thought that 50 to $60 \mathrm{deg}$. is the best fiber angle for redesigned Zig-Zag chair with fold structure.

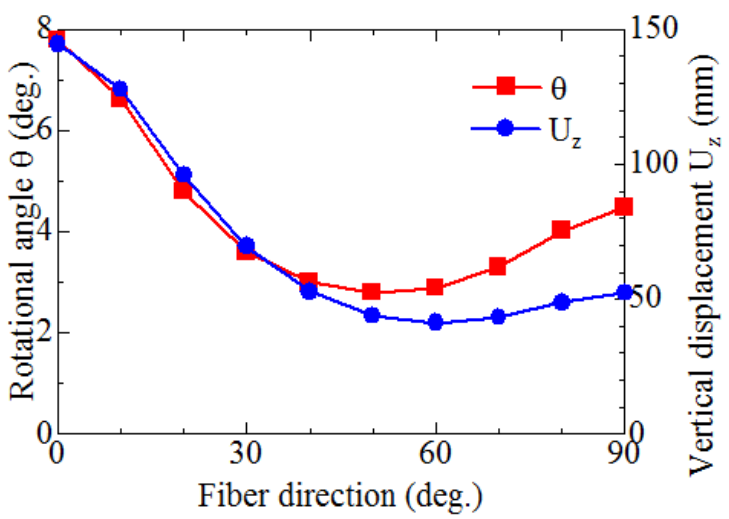

Figure 17: Rotational angle and vertical displacement changing fiber angle in CFRP cloth. 


\section{Conclusions}

In this study, widely used composite material CFRP which has very high specific strength and elastic modulus with high anisotropic was targeted. We examined its material characteristic and pursued the optimum structure design effectively by using its material characteristic, and developed a new product design method integrated with strength evaluation and styling. As a subject of study, using ZigZag chair redesigned by Haruhiko Iida in 2015, we pursued the optimum shape and strength design made of CFRP.

The results obtained in this study are summarized as follows.

1) We proposed a simplified modeling method on CAD to represent the characteristics of CFRP which has high anisotropy, and it can well represent the CFRP characteristics in CAE analysis.

2) By using the modeling method, the behavior of CFRP structure can be well represented, and we can estimate strength and stiffness of CFRP structure by simulation.

3) We proposed several methods to improve torsional rigidity of redesigned ZigZag chair made of CFRP, using box structure in leg part and changing the fiber direction of cloth, and changing fiber angle of cloth mesh, and confirmed its effectiveness.

As future works, we will modify the model of a Zig-Zag chair of excellent shape shown in Fig. 9, and will improve its rigidity sufficiently.

Also, we pursue the shape and structure that enhance its original design quality.

\section{References}

[1] Matsuoka Y. \& Miyamoto S., The concept of optimal design, Kyoritsu Shuppan Co., Ltd. (in Japanese), 2008.

[2] Strunk Jr. W. \& White E. B., The Elements of Style, third ed., Macmillan, New York, 1979.

[3] Iida H., Sakamoto H. \& Ohbuchi Y., Development and application of new design method by high-strength composite material, Applied Mechanics and Materials, 372, pp. 17-20, 2013.

[4] Iida H., Sakamoto H., Ohbuchi Y. \& Fujishima T., Application of new design method by high-strength composite material, Applied Mechanics and Materials, 607, pp. 915-919, 2014.

[5] Iida H., Ohbuchi Y., Fujishima T. \& Sakamoto H., Application and Evaluation of New Design Method by using Carbon Fiber-Reinforced Plastics, Applied Mechanics and Materials Vol. 749, pp. 231-235, 2015.

[6] Iida H., Sakamoto H., Ohbuchi Y. and Fujishima T., Application and verification of new design method in high strength composite material, Journal of JSME 81, No.824, pp. 1-8, (in Japanese), 2015.

[7] Ohbuchi Y., Iida H., Katayama T., Tanaka K., Nakayama Y. \& Sakamoto H., Redesign of ZIGZAG Chair by fiber reinforced plastics -fusing of product design and engineering, WIT Transactions on Modelling and Simulation, Vol. 59, pp. 25-32, 2015. 